ISSN 0103-5150

Fisioter. Mov., Curitiba, v. 25, n. 3, p. 649-658, jul./set. 2012 Licenciado sob uma Licença Creative Commons

\title{
Efeito do treinamento concorrente nos componentes da síndrome metabólica de homens de meia-idade
}

\author{
Effect of concurrent training on components of the \\ metabolic syndrome in middle-aged men
}

\section{Giovana Vergínia de Souza ${ }^{[a]}$, Cleiton Augusto Libardi ${ }^{[b]}$, José Rocha Jr. ${ }^{[c]}$, Vera Aparecida Madruga ${ }^{[\mathrm{d}]}$, Mara Patrícia Traina Chacon-Mikahil ${ }^{[\mathrm{e}]}$}

[a] Doutoranda em Atividade Física Adaptada pela Faculdade de Educação Física da Universidade de Campinas (UNICAMP), Campinas, SP - Brasil, e-mail: gigia@fef.unicamp.br

[b] Pós-doutorando em Educação Física pela Faculdade de Educação Física e Esporte da Universidade de São Paulo (USP), pesquisador colaborador da Faculdade de Educação Física da Universidade Estadual de Campinas (UNICAMP), Campinas, SP - Brasil, e-mail: cleiton.libardi@hotmail.com

[c] Doutor em Clínica Médica pela Universidade Estadual de Campinas (UNICAMP), docente da disciplina de Cardiologia da Faculdade de Ciências Médicas da UNICAMP, Campinas, SP - Brasil, e-mail: jrocha@fcm.unicamp.br

[d] Doutora em Educação Física pela Universidade Estadual de Campinas (UNICAMP), docente do Programa de PósGraduação em Educação Física da Faculdade de Educação Física da Universidade Estadual de Campinas (UNICAMP), Campinas, SP - Brasil, e-mail: madruga@fef.unicamp.br

[e] Doutora em Educação Física pela Universidade Estadual de Campinas (UNICAMP), docente do Programa de PósGraduação em Educação Física da UNICAMP, Campinas, SP - Brasil, e-mail: marapatricia@fef.unicamp.br

\section{Resumo}

Introdução: A prática regular de exercícios físicos é considerada fundamental para prevenção e tratamento da Síndrome Metabólica, porém pouco se sabe sobre a forma ideal de treinamento. Objetivo: Verificar o efeito de 16 semanas de treinamento concorrente (TC) com frequência semanal e duração da sessão similar ao treinamento de força (TF) e treinamento aeróbio (TA) isolados sobre os componentes da síndrome metabólica (SM) de homens de meia-idade. Métodos: Foram selecionados 42 homens clinicamente saudáveis distribuídos em quatro grupos: TA $(n=13)$, TF $(n=9)$, TC $(n=10)$ e grupo controle $(G C, n=10)$. Os treinamentos foram compostos por duas etapas (E1 e E2) com duração de oito semanas cada, e frequência de três sessões/semana (TF: dez exercícios com 3 x 8-10 RM; TA: 60 min. de caminhada ou corrida a 55-85\% VO2 $2_{\text {pico }}$ TC: seis exercícios com 3 x 8-10 RM, seguidos de 30 min. de caminhada ou corrida a 55-85\% $\mathrm{VO}_{2 \text { pico }}$ ). 
Foram avaliadas circunferência de cintura (CC), perfil lipídico, glicose, pressão arterial (PA) e força máxima e $\mathrm{VO}_{2 \text { pico }}$. Resultados: A CC reduziu para o TA $(-1,70 \% ; \mathrm{p}=0,023)$ e TC $(-1,66 \% ; \mathrm{p}=0,018)$ e o LDL para o TF $(-25,03 \% ; p=0,047)$ e TC $(29,74 \% ; p=0,011)$. 0 TC promoveu redução na PA sistólica $(-7,83 \% ; p=0,029)$. A força máxima e $\mathrm{VO}_{2 \text { pico }}$ aumentaram para os três grupos de treinamento $(\mathrm{p}>0,05)$. Conclusão: A realização do TC frequência semanal e duração das sessões similar ao TA e TF isolados é mais eficaz para a melhora de componentes da SM.

Palavras-chave: Perfil lipídico. Pressão arterial. Treinamento aeróbio. Treinamento de força.

\section{Abstract}

Introduction: The practice of regular physical exercise is considered critical to prevention and treatment of metabolic syndrome, but little is known about the ideal form of training. Objective: To investigate the effect of 16 weeks of concurrent training (CT) with weekly frequency and duration of the session similar to strength training (ST) and endurance training (ET) isolates, in the components of metabolic syndrome (MS) of middle-age men. Methods: Forty-two clinically healthy men were selected and divided into four groups: ET ( $n=13), S T(n=9), C T(n=10)$ and control group (CG, $n=10)$. The training was composed of two steps (E1 and E2) with a duration of eight weeks each, and frequency of three sessions/week (ST: ten exercises with $3 \times 8-10$ RM; AT: 60 min. Walking or running at $55-85 \% V_{2 p e a k}$ CT: six exercises with $3 \times$ RM 8.10, followed by 30 min. walking or running at $55-85 \% V_{2 \text { peak }}$. We evaluated waist circumference (WC), lipid profile, glucose, blood pressure (BP), maximum force and $V O_{2 p e a k}$ Results: The WC reduced to the AT $(-1.70 \%, p=0.023)$ and CT $(-1.66 \%, p=0.018)$ and LDL to the ST $(-25.03 \%, P=0.047)$ and CT $(29.74 \%, p=$ $0.011)$. The CT promoted the reduction in systolic $B P(-7.83 \%, P=0.029)$. The maximum force and peak $V_{2}$ increased for the three training groups ( $p>0.05$ ). Conclusion: The performance of CT weekly frequency and duration of sessions similar to the ET and ST alone is more effective for the improvement of MS components.

Keywords: Lipid profile. Blood pressure. Endurance training. Strength training.

\section{Introdução}

A Organização Mundial de Saúde (OMS) estima que, em 2015, mais de 1,5 bilhão de pessoas no mundo apresentarão sobrepeso (IMC $\geq 25 \mathrm{~kg} / \mathrm{m}^{2}$ ) ou obesidade (IMC $\geq 30 \mathrm{~kg} / \mathrm{m}^{2}$ ). 0 sobrepeso e a obesidade são resultado do aumento do sedentarismo e da alta ingestão calórica (1), o que contribui para o desenvolvimento da Síndrome Metabólica (SM) (2), que, por sua vez, aumenta a possibilidade do desenvolvimento de diabetes mellitus tipo 2 e de doença cardiovascular (DCV) (3).

A prática regular de exercícios físicos é considerada fundamental para prevenção e tratamento da SM $(4,5)$. Portanto, tem-se sugerido que um regime ótimo de treinamento para os indivíduos que exibem os fatores de risco cardiovascular associados a SM requer a associação do treinamento aeróbio (TA) ao treinamento de força (TF) (6). De fato, importantes diretrizes têm preconizado a realização do treinamento concorrente (TC; i. e. TF e TA em uma mesma unidade de treinamento ou em dias alternados) durante o envelhecimento $(7,8)$.

Para que o TA e o TF (i. e. TC) sejam realizados, é necessário aumentar o volume do TC quando comparada a realização do TA e do TF de forma isolada. Stewart et al. (9) demonstraram que três sessões semanais de TC melhoram os componentes da SM de indivíduos de meia-idade e idosos, entretanto, o protocolo utilizado foi de aproximadamente duas horas a sessão. Por outro lado, quando a duração da sessão é menor, para que os mesmos benefícios sejam obtidos, parece necessário aumentar a frequência de treinamento (i. e. 2x TA e 2x TF) (10), sendo o dobro das sessões de TA e TF isoladas (i. e. 2x). Dessa forma, é possível que a vantagem do TC para a melhora dos componentes da SM deva-se ao maior volume de treinamento em relação à realização do TA e TF de forma isolada. Embora a redução dos componentes da SM ocorra quando um elevado volume do TC é realizado, duração das sessões e frequência semanal de TC 
reduzidas podem aumentar a aderência (11), bem como estimular o início da prática de exercícios físicos por pessoas sedentárias. Porém, ainda não se sabe se o TC realizado com frequência semanal e duração da sessão similar ao TA e o TF isolados continua apresentando vantagens para a melhora dos componentes da SM em relação a esses regimes de treinamento.

Dessa forma, o objetivo do presente estudo é verificar o efeito do TC com frequência semanal e duração das sessões similares ao TA e TF nos componentes da SM de homens de meia-idade.

\section{Métodos}

\section{Amostra}

Participaram do estudo 42 homens, com idade entre 40 e 60 anos. Todos foram previamente informados sobre os métodos do estudo e, ao concordarem em participar dele, assinaram o termo de consentimento livre e esclarecido. 0 presente estudo foi aprovado pelo Comitê de Ética em Pesquisa da Universidade.

Como critérios iniciais de inclusão, os voluntários deveriam ser considerados irregularmente ativos $\mathrm{B}$ ou sedentários, segundo o questionário internacional de nível de atividade física (IPAQ) (12), e não ter participado regularmente de nenhum programa de treinamento físico nos últimos seis meses precedentes ao estudo, segundo o questionário de atividade física habitual "Baecke" (13). Todos os voluntários realizaram avaliação clínica composta de eletrocardiograma de repouso e teste ergométrico, conduzidos por um médico cardiologista, antes do início do estudo. Como critérios de exclusão foram adotados a manifestação de doença isquêmica do miocárdio, arritmias, hipertensão arterial, diabetes mellitus e obesidade mórbida. Somente fizeram parte da amostra do presente estudo os voluntários que participaram de pelo menos $90 \%$ das sessões de treinamento realizadas, e que não se ausentaram por mais de duas sessões de treinamento consecutivas.

\section{Delineamento experimental}

As avaliações antropométricas, do perfil lipídico, glicose plasmática, pressão arterial, força muscular e cardiorrespiratória foram realizadas antes (pré), e ao fim (pós) do período experimental. Os voluntários foram distribuídos em quatro grupos: TA, $n=13,46,8$ $\pm 3,4$ anos; $T F, n=9,48,7 \pm 5,5$ anos; $T C, n=10,47,5$ $\pm 5,1$ anos; $\mathrm{GC}, \mathrm{n}=10,49 \pm 5,3$ anos.

Os grupos TF, TA e TC foram submetidos a 16 semanas de treinamento, enquanto o GC permaneceu por todo o período experimental sem realizar nenhum treinamento físico. Os protocolos de TF, TA e TC foram divididos em etapa 1 (E1) e etapa 2 (E2), com duração de oito semanas consecutivas cada, em que os voluntários realizaram três sessões semanais de treinamento, em dias alternados (segundas, quartas e sextas-feiras). Entre as duas etapas houve um intervalo de uma semana sem treinamento, para realização das avaliações cardiorrespiratórias. A partir dos resultados obtidos na avaliação cardiorrespiratória, foi realizado ajuste na intensidade de caminhada e corrida dos protocolos TA e TC para E2. A duração das sessões de TA, TF e TC foi de aproximadamente 60 minutos. Os programas de treinamento foram descritos detalhadamente $(14,15)$.

\section{Antropometria}

A avaliação da massa corporal foi realizada por meio de balança de plataforma, Filizola ${ }^{\circledR}$ digital modelo ID-1500 (precisão de 0,1 kg) e a estatura foi obtida em um estadiômetro de madeira com precisão de $0,1 \mathrm{~cm}(16)$. A partir das medidas de massa corporal e estatura, calculou-se o índice de massa corpórea (IMC), por meio do quociente peso corporal/estatura (2). Foi avaliada, também, a circunferência de cintura (CC), por meio de fita flexível (17).

\section{Perfil lipídico e glicose plasmática}

Amostras de sangue $(\sim 10 \mathrm{ml})$ foram obtidas da veia anticubital pela manhã, após $12 \mathrm{~h}$ de jejum, antes e após o período. As concentrações séricas de triglicerídeos (TG), lipoproteínas de alta densidade (HDL) e glicose foram mensuradas por meio de um analisador automático (Technicon, RA 1000, USA) e kits comercialmente disponíveis (Laborlab, Brazil). A lipoproteína de baixa densidade (LDL) foi calculada de acordo com a equação de Friedwald (18).

\section{Pressão arterial}

Ao chegarem ao laboratório, os voluntários permaneceram sentados por cinco minutos. Em seguida, 
a pressão arterial foi aferida por meio do método auscultatório, utilizando-se de um esfigmomanometro de coluna de mercúrio (Narcosul, Brazil). As medidas foram realizadas em dois dias consecutivos. A média dos valores obtidos foi utilizada para o estudo (19).

\section{Avaliação da força muscular}

A força muscular foi mensurada por meio do teste de uma repetição máxima (1-RM) nos exercícios supino reto, leg press e flexão dos cotovelos (20). Todos os exercícios foram precedidos por uma série de aquecimento de dez repetições, com aproximadamente $50 \%$ da carga prevista para a primeira tentativa de cada teste de 1-RM, com os testes sendo iniciados três minutos após o aquecimento. Em seguida, os voluntários foram orientados a realizar uma única repetição com a carga prevista para 1-RM. Caso fosse completada, ou mesmo se não fosse, uma segunda tentativa seria realizada após um intervalo de três a cinco minutos, com carga (kg) superior ou inferior à empregada anteriormente. Uma terceira e última tentativa seria realizada se ainda não houvesse determinado a carga correspondente a uma única repetição máxima. Todos os voluntários realizaram duas sessões de testes com intervalo de $48 \mathrm{~h}$ entre elas, visando à familiarizar-se com eles e, assim, minimizar os efeitos de aprendizagem. Foram consideradas para análise as cargas obtidas na segunda sessão.

\section{Avaliação cardiorrespiratória}

Os voluntários executaram um protocolo de teste em esteira ergométrica (Quinton TM55, Bothell, Washington, EUA), em que as trocas gasosas foram coletadas continuamente, respiração a respiração, por meio de um sistema metabólico de análise de gases (CPX, Medical Graphics, St, Paul, Minnesota, USA). 0 protocolo consistiu de uma velocidade inicial de aquecimento de $4 \mathrm{~km} / \mathrm{h}$ por 2 minutos, seguidos de acréscimos de $0,3 \mathrm{~km} / \mathrm{h}$ a cada $30 \mathrm{~s}$, com inclinação constante de 1\% (21) até a exaustão física. A isso, seguia-se então um período de 4 minutos de recuperação, sendo o primeiro minuto a $5 \mathrm{~km} / \mathrm{h}$, reduzindo-se $1 \mathrm{~km} / \mathrm{h}$ a cada minuto.
Limiar ventilatório (LV) e ponto de compensação respiratória (PCR), consumo oxigênio pico $\left(\mathrm{VO}_{2 \text { pico }}\right)$

O LV e o PCR foram determinados por meio de uma análise visual gráfica (22), realizada por três observadores previamente treinados, e familiarizados. $0 \mathrm{VO}_{2 \text { pico }}$ foi expresso pela média dos valores nos últimos 30 segundos da avaliação cardiorrespiratória (23). Para confirmar a ocorrência do $\mathrm{VO}_{\text {2pico' }}$ deveriam ser observados pelo menos dois dos três critérios a seguir: (1) um platô no $\mathrm{VO}_{2}$, ou seja, nenhuma ou pouca variação no $\mathrm{VO}_{2}(<2,1 \mathrm{~mL}, \mathrm{~kg}-1$, min-1), apesar do aumento na intensidade do exercício; (2) razão de trocas respiratórias maior que 1,10; (3) frequência cardíaca (FC) maior que $90 \%$ do máximo predito para a idade (24).

\section{Análise estatística}

Inicialmente foi realizado o teste de ShapiroWilk para testar a normalidade dos dados. Em seguida, foi realizada a análise de variância para medidas repetidas (two-way ANOVA) para comparação inter e intragrupos. Para comparação entre as diferenças percentuais, foi utilizada a análise de variância de um caminho (one-way ANOVA). 0 teste post hoc de Newman-Keuls foi empregado para a identificação das diferenças específicas nas variáveis em que os valores de $\mathrm{F}$ encontrados foram superiores ao do critério de significância estatística estabelecida $(p<0,05)$. Para todas as análises, foi utilizado o pacote estatístico Statistica ${ }^{\circledR} 6.1$ (StatSoft Inc.,Tulsa, OK). Os dados são apresentados em média e desvio-padrão.

\section{Resultados}

\section{Antropometria}

Não foram verificadas alterações significantes $(p>0,05)$ na massa corporal e IMC em nenhum dos grupos estudados. A CC reduziu significantemente para o TA $(-1,70 \% ; \mathrm{p}=0,023)$ e $\mathrm{TC}(-1,66 \% ; \mathrm{p}=$ $0,018)$ após 16 semanas de treinamento, sem diferença no percentual de modificação entre os grupos (Tabela 1). 
Tabela 1- Variáveis antropométricas pré e pós 16 semanas de treinamento aeróbio (TA, $n=13)$, treinamento de força (TF, $n=9$ ), treinamento concorrente (TC, $n=10)$ e grupo controle (GC, $n=10)$

\begin{tabular}{lccccc}
\hline Variáveis & & TA & TF & TC & GC \\
\hline Estatura $(\mathbf{m})$ & & $1,75 \pm 0,05$ & $1,71 \pm 0,05$ & $1,72 \pm 0,05$ & $1,72 \pm 0,05$ \\
& Pré & $79,4 \pm 8,6$ & $77,8 \pm 13,2$ & $82,6 \pm 8,8$ & $73,5 \pm 6,1$ \\
MC(kg) & Pós & $79,3 \pm 7,8$ & $77,4 \pm 12,5$ & $82,4 \pm 9,1$ & $73,4 \pm 6,2$ \\
& $\Delta \%$ & 0,08 & 0,59 & $-0,27$ & $-0,17$ \\
& Pré & $25,6 \pm 2,0$ & $26,1 \pm 3,5$ & $27,6 \pm 2,4$ & $24,9 \pm 2,0$ \\
IMC $\left(\mathbf{k g} / \mathbf{m}^{2}\right)$ & Pós & $25,6 \pm 1,8$ & $26,2 \pm 3,0$ & $27,5 \pm 2,7$ & $24,8 \pm 2,0$ \\
& $\Delta \%$ & $-0,03$ & 0,36 & $-0,32$ & $-0,24$ \\
\hline
\end{tabular}

Fonte: Dados da pesquisa.

Legenda: MC - massa corporal; IMC - índice de massa corporal; $\Delta \%$ - Percentual de modificação.

Perfil lipídico e glicose plasmática

Os resultados do perfil lipídico (Tabela 2) demonstraram reduções significantes no LDL para o TF $(-25,03 \% ; p=0,047)$ e TC $(29,74 \% ; p=0,011)$, sem alterações para o TA e GC (p > 0,05). 0 HDL e TG não apresentaram alterações significantes ( $p$ > $0,05)$ para nenhum dos grupos estudados. Já a glicose plasmática apresentou aumento significante no GC (20,61\%; $p=0,0001)$, sem alterações para os demais grupos (Tabela 2).

\section{Pressão arterial}

Somente o TC apresentou redução significante na PAS após 16 semanas de intervenção $(-7,83 \%$; $p=0,029)$. Já a PAD não apresentou alterações significantes para os grupos estudados $(p>0,05)$.

\section{Componentes da síndrome metabólica}

A Figura 1 demonstra o número de componentes da síndrome metabólica dos grupos TA, TF, TC e GC. Somente o TC apresentou reduções do número de componentes da SM após 16 semanas $(p=0,044)$.

\section{Consumo de oxigênio pico $\left(\mathrm{VO}_{2 \text { pico }}\right)$}

A maioria dos voluntários do presente estudo não apresentou platô no $\mathrm{VO}_{2}$, portanto, foi adotado como critério de potência aeróbia máxima, o $\mathrm{VO}_{2 \text { pico }}$

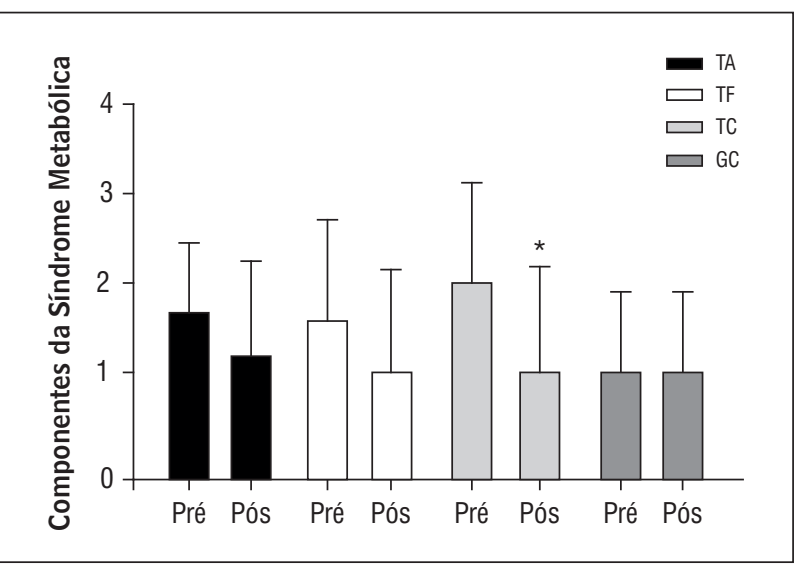

Figura 1 - Componentes da síndrome metabólica Pré e Pós 16 semanas de treinamento aeróbio (TA), de força (TF), concorrente (TC) e grupo controle (GC) $(p<0,05)$; *Diferença significante em comparado ao pré-treinamento $(p<0,05)$

(24). A Tabela 3 apresenta os resultados do $\mathrm{VO}_{2 \text { pico }}$ pré e pós intervenção. Foi observado aumento significante no $\mathrm{VO}_{2 \text { pico }}$ para o TA $(19,48 \% ; \mathrm{p}=0,0001)$, TF $(8,61 \% ; p=0,042)$ e TC $(11,54 \% ; p=0,048)$. Na comparação entre os grupos, verificou-se que o TA apresentou maior percentual de modificação no $\mathrm{VO}_{2 \text { pico }}(\mathrm{p}=0,003)$, quando comparado ao TF e TC.

\section{Força máxima (1-RM)}

A força máxima aumentou significantemente no leg press para o $\mathrm{TA}(33,97 \% ; \mathrm{p}=0,0002), \mathrm{TF}(38,11 \%$; $\mathrm{p}=0,0001)$ e TC $(34,74 \% ; \mathrm{p}=0,0001)$, sem diferenças significantes entre os grupos $(p>0,05)$. 0 mesmo 
Tabela 2 - Componentes da síndrome metabólica pré e pós 16 semanas de treinamento aeróbio (TA, $n=13)$, treinamento de força (TF, $n=9)$, treinamento concorrente (TC, $n=10)$ e grupo controle (GC, $n=10)$

\begin{tabular}{lccccc}
\hline Variáveis & & TA & TF & TC & GC \\
\hline \multirow{4}{*}{ CC (cm) } & Pré & $89,30 \pm 8,00$ & $90,60 \pm 10,00$ & $95,40 \pm 5,20$ & $86,10 \pm 6,10$ \\
& Pós & $87,80 \pm 7,60^{*}$ & $89,50 \pm 10,20$ & $93,80 \pm 4,90^{\star}$ & $86,50 \pm 6,20$ \\
& $\Delta \%$ & $-1,70$ & $-1,26$ & $-1,66$ & 0,46 \\
& Pré & $114.07 \pm 33.51$ & $144.75 \pm 46.04$ & $152.95 \pm 64.87$ & $128.18 \pm 36.79$ \\
LDL (mg/dL) & Pós & $125.35 \pm 31.47$ & $108.51 \pm 44.18^{*}$ & $107.45 \pm 41.14^{*}$ & $118.5 \pm 40.04$ \\
& $\Delta \%$ & 9.89 & -25.03 & -29.74 & -7.55 \\
& Pré & $38,85 \pm 11,02$ & $47,17 \pm 9,65$ & $52,28 \pm 19,20$ & $44,62 \pm 14,63$ \\
HDL (mg/dL) & Pós & $41,92 \pm 11,52$ & $52,39 \pm 7,20$ & $48,86 \pm 9,05$ & $43,40 \pm 13,83$ \\
& $\Delta \%$ & 7,90 & 11,07 & $-6,54$ & $-2,74$ \\
& Pré & $159,61 \pm 99,59$ & $156,76 \pm 70,57$ & $192,83 \pm 111,05$ & $138,05 \pm 80,95$ \\
TG (mg/dL) & Pós & $148,38 \pm 95,09$ & $146,46 \pm 41,84$ & $130,71 \pm 51,08$ & $152,20 \pm 84,26$ \\
& $\Delta \%$ & $-7,03$ & $-6,56$ & $-32,21$ & 10,24 \\
& Pré & $94,30 \pm 10,91$ & $90,09 \pm 13,48$ & $95,46 \pm 11,18$ & $80,25 \pm 7,62$ \\
Glicose (mg/dL) & Pós & $95,30 \pm 7,54$ & $92,66 \pm 5,80$ & $89,38 \pm 6,34$ & $96,80 \pm 5,75 *$ \\
& $\Delta \%$ & 1,06 & 2,85 & $-6,36$ & 20,61 \\
& Pré & $114,61 \pm 12,14$ & $121,11 \pm 12,61$ & $127,60 \pm 12,24$ & $118,80 \pm 15,80$ \\
PAS (mmHg) & Pós & $117,30 \pm 7,01$ & $115,11 \pm 8,37$ & $117,60 \pm 9,22^{*}$ & $114,60 \pm 8,89$ \\
& $\Delta \%$ & 2,34 & $-4,95$ & $-7,83$ & $-3,53$ \\
& Pré & $80,46 \pm 6,59$ & $82,44 \pm 7,33$ & $86,00 \pm 6,64$ & $83,40 \pm 11,66$ \\
PAD (mmHg) & Pós & $82,46 \pm 4,90$ & $82,00 \pm 9,74$ & $87,20 \pm 4,73$ & $80,70 \pm 4,05$ \\
& $\Delta \%$ & 2,48 & $-0,53$ & 1,39 & $-3,23$ \\
\hline
\end{tabular}

Fonte: Dados da pesquisa.

Legenda: $\Delta \%$ - Percentual de modificação; * Diferença significante comparado ao pré-treinamento $(p<0,05)$.

Tabela 3 - Consumo de oxigênio pico e força máxima pré e pós 16 semanas de treinamento aeróbio (TA, $n=13$ ), treinamento de força (TF, $n=9$ ), treinamento concorrente (TC, $n=10$ ) e grupo controle (GC, $n=10$ )

\begin{tabular}{|c|c|c|c|c|c|}
\hline Variáveis & & TA & $\mathrm{TF}$ & TC & GC \\
\hline \multirow{3}{*}{$\mathrm{VO}_{2 \text { pico }}(\mathrm{ml} / \mathrm{kg} / \mathrm{min}$.) } & Pré & $31,74 \pm 4,36$ & $34,02 \pm 4,64$ & $30,52 \pm 6,16$ & $31,11 \pm 5,41$ \\
\hline & Pós & $37,93 \pm 3,45^{\star}$ & $36,95 \pm 3,38^{\star}$ & $34,04 \pm 5,82^{*}$ & $30,55 \pm 3,84$ \\
\hline & $\Delta \%$ & 19,48 & $8,61 \ddagger$ & 11,54 & $-1,80 \ddagger$ \\
\hline \multirow{3}{*}{ Leg press (kg) } & Pré & $197,00 \pm 41,47$ & $245,30 \pm 37,13$ & $233,40 \pm 39,20$ & $221,41 \pm 49,70$ \\
\hline & Pós & $263,92 \pm 62,5^{\star}$ & $338,80 \pm 34,7^{*}$ & $314,50 \pm 66,3^{*}$ & $218,66 \pm 60,49$ \\
\hline & $\Delta \%$ & $33,97 \dagger$ & $38,11 \dagger$ & $34,74 \dagger$ & $-1,24$ \\
\hline \multirow{3}{*}{ Supino reto (kg) } & Pré & $62,66 \pm 10,62$ & $78,80 \pm 5,43$ & $72,75 \pm 9,69$ & $62,18 \pm 10,41$ \\
\hline & Pós & $62,75 \pm 11,54$ & $94,50 \pm 6,94^{*}$ & $87,08 \pm 10,68 *$ & $61,27 \pm 11,46$ \\
\hline & $\Delta \%$ & 0,13 & $19,92 \dagger \ddagger$ & $19,70 \dagger \ddagger$ & $-1,46$ \\
\hline
\end{tabular}

Fonte: Dados da pesquisa.

Legenda: Diferença significante comparado ao pré-treinamento $(p<0,05)$; $\uparrow$ Diferença significante comparado ao grupo controle $(p<0,05)$; ‡ Diferença significante comparado ao treinamento aeróbio $(p<0,05) ; \Delta \%$ - Percentual de modificação. 
ocorreu para o supino reto, porém somente para o TF $(19,92 \% ; p=0,0001)$ e TC $(19,70 \% ; p=0,0001)$, sem diferenças significantes entre eles ( $p>0,05)$ (Tabela 3$)$.

\section{Discussão}

O objetivo do presente estudo foi verificar o efeito do TC com frequência semanal e duração das sessões similar ao TA e TF, nos componentes da SM de homens de meia-idade. Os principais achados demonstraram que o TC com duração de aproximadamente $60 \mathrm{~min}$. (i. e. $\sim 30 \mathrm{~min}$. TF e $\sim 30 \mathrm{~min}$. TA) promoveu melhora em mais componentes da SM, comparado ao TA ( $\sim 60 \mathrm{~min}$.) e TF ( $\sim 60 \mathrm{~min}$.) isolados, pois apresentou reduções na CC, LDL e PAS. 0 TF, por sua vez, apresentou reduções somente no LDL, enquanto que o grupo TA reduziu somente a CC. Além disso, todos os grupos apresentaram aumento da força muscular e aptidão cardiorrespiratória.

0 aumento da força muscular e da aptidão cardiorrespiratória é de grande importância para a população envelhecendo (7). No presente estudo, embora todos os grupos de treinamento tenham apresentado aumento na força muscular e na aptidão aeróbia, o TA apresentou maior aumento no $\mathrm{VO}_{\text {2pico }}$ comparado aos demais grupos, provavelmente por ter realizado o dobro do volume de exercícios de caminhada/corrida (i. e. $60 \mathrm{~min}$.), quando comparado ao TC (30 min.). Já em relação aos ganhos de força muscular, para os membros inferiores, os resultados foram similares entre $\mathrm{TF}$, TA e TC, mesmo o TA não tendo realizado exercícios específico para o desenvolvimento dessa capacidade. Contrariando nossos achados, Sillanpää et al. (25) não verificaram aumento do $\mathrm{VO}_{2 \text { máx }}$ após o $\mathrm{TF}$, embora em relação à força muscular seja mais comum encontrar melhoras na força de membros inferiores realizando somente TA $(9,26,27)$. É possível que, em virtude do baixo nível de aptidão física dos indivíduos de meia-idade do presente estudo, foi possível observar aumento da força de membros inferiores mesmo com o TA.

0 aumento da força muscular e aptidão cardiorrespiratória apresentado nos grupos estudados parecem ser importantes para evitar o desenvolvimento da SM. Demonstrou-se que indivíduos com maior aptidão cardiorrespiratória possuem menor probabilidade de desenvolver doenças crônico-degenerativas, bem como a força muscular parece ser inversamente proporcional à SM. Indivíduos com maior força possuem
34\% menos riscos de desenvolver SM (28). Assim, sugere-se que o TA e TF sejam associados (i. e. TC) para a redução dos fatores de risco de doenças cardiovascular em homens de meia-idade. Além disso, parecem fornecer diferentes benefícios (6). Porém, estudos com TC têm demonstrado que a duração da sessão ou a frequência semanal são aumentadas em relação ao TA e ao TF realizados de forma isolada $(9,10)$, o que pode prejudicar a adesão de indivíduos sedentários a programas de exercícios físicos (11). 0 presente estudo realizou o TC com frequência semanal e duração da sessão similar ao TA e ao TF isolados e menor que outros estudos que investigaram os efeitos do TC na SM $(9,10)$ e, mesmo assim, apresentou vantagens em relação ao TA e ao TF.

Um dos componentes que apresentou melhora significante com o TC foi a CC. Estudos têm demonstrado que a CC tem forte correlação com a gordura visceral (29) e que esta é um importante preditor de alterações metabólicas. 0 acúmulo de gordura visceral aumenta a lipólise e, consequentemente, a liberação de ácidos graxos livres (AGL), resultando em diminuição dos transportadores de glicose (GLUT4) e conduzindo, assim, a um quadro resistência a insulina (30). 0 presente estudo demonstrou redução da CC para o TC e TA $(-1,70 \%$ e $-1,66 \%$, respectivamente), possivelmente pelo aumento da utilização de AGL durante o exercício, visto que o predomínio aeróbio desses regimes de treinamento é maior que o TF. Entretanto, a redução da CC não refletiu em alterações nos níveis de glicose plasmática em nenhum dos grupos estudados. Vale ressaltar que os voluntários dos três grupos investigados eram normoglicêmicos, o que pode estar relacionado ao fato de a glicemia não ter sido alterada.

Os grupos TC, juntamente com o TF, demonstraram diminuição do colesterol LDL corroborando, assim, com outros achados da literatura $(31,32)$. Por outro lado, alguns estudos não têm demonstrado alterações com o TF (33). Sugere-se que alterações no colesterol LDL com TF ocorram somente quando este é realizado com intensidades acima de $70 \%$ de 1-RM, por períodos maiores que 12 semanas (34). No presente, a intensidade realizada foi de moderada para alta (i. e. 8-10 RM) durante 16 semanas, podendo assim ter contribuído para as reduções no LDL no grupo TF.

Outro importante componente da SM é a pressão arterial (PA). A manutenção da PA, principalmente na meia-idade, torna-se importante, tendo em vista 
que o aumento de $20 \mathrm{mmHg}$ na PA sistólica (PAS) e de $10 \mathrm{mmHg}$ na PA diastólica (PAD) dobra o risco de morte nessa faixa etária (35). Porém, a redução PAS e PAD de repouso, por meio do exercício físico, parece mais pronunciada em hipertensos $(6,9 / 4,9$ mmHg) em relação a normotensos $(1,9 / 1,6 \mathrm{mmHg})$ (36). No presente, somente o TC apresentou reduções significantes na PA, especificamente na PAS (7,83\%), embora o TA seja apontado como o regime de treinamento mais recomendado para reduções na PA (37). A associação do TA com o TF (i. e. TC) no presente estudo, demonstrou ser mais eficaz que o TA isolado, mesmo este apresentando um volume maior de exercícios aeróbios (60 min.) quando comparado ao TC (30 min.). Recentemente, foi demonstrado que duas séries de seis exercícios realizados a $80 \%$ de 1-RM, seguidos de 30 minutos de exercício em cicloergômetro em três diferentes intensidades (50, 65 e $80 \%$ do $\mathrm{VO}_{2 \text { pico }}$ ), promoveram hipotensão pós-exercício, sendo mais pronunciada a $65 \%$ do $\mathrm{VO}_{2 \text { pico }}$ (36). Durante o presente estudo, embora a intensidade nos 30 minutos TA no TC tenha variado entre $50-85 \% \mathrm{VO}_{2 \text { ico' }}$ os indivíduos permaneceram mais tempo na zona do limiar ventilatório (entre $60-70 \%$ $\mathrm{VO}_{2 \text { pico }}$ ), que pode explicar a redução da PAS no TC.

\section{Conclusões}

O presente estudo demonstrou que diferentes regimes de treinamento podem contribuir para a melhora de componentes da SM. Entretanto, o TC, com volume (i. e. frequência semanal e duração da sessão) similar ao do TA e do TF e menor que o da maioria dos estudos que investigaram os efeitos do TC na SM $(10,25,28,38-40)$, foi mais eficaz para a melhora dos componentes da SM. Dessa forma, a realização do TC três vezes por semana, com duração das sessões de aproximadamente 60 minutos, pode contribuir para a redução de componentes da SM e prevenção de doenças cardiovasculares.

\section{Agradecimentos}

Agradecemos ao Conselho Nacional de Desenvolvimento Científico e Tecnológico (CNPq) pelo apoio financeiro a este estudo, à Disciplina de Cardiologia do Departamento de Clínica Médica FCM/Unicamp e ao Projeto de Extensão Musculação FEF/Unicamp.

\section{Referência}

1. Morabia A, Abel T. The WHO report "preventing chronic diseases: a vital investment" and us. Soz Praventivmed. 2006;51(2):74. doi:10.1007/s00038-005-0015-7.

2. Guilbert JJ. The world health report 2002 - reducing risks, promoting healthy life. Educ Health (Abingdon). 2003;16(2):230. doi:10.1080/1357628031000116808.

3. Lakka HM, Laaksonen DE, Lakka TA, Niskanen LK, Kumpusalo E, Tuomilehto J, et al. The metabolic syndrome and total and cardiovascular disease mortality in middle-aged men. JAMA. 2002;288(21):2709-16. doi:10.1001/jama.288.21.2709.

4. Lakka TA, Laaksonen DE, Lakka HM, Mannikko N, Niskanen LK, Rauramaa R, et al. Sedentary lifestyle, poor cardiorespiratory fitness, and the metabolic syndrome. Med Sci Sports Exerc. 2003;35(8):1279-86. doi:10.1249/01.MSS.0000079076.74931.9A.

5. Laaksonen DE, Niskanen L, Nyyssonen K, Punnonen K, Tuomainen TP, Valkonen VP, et al. C-reactive protein and the development of the metabolic syndrome and diabetes in middle-aged men. Diabetologia. 2004;47(8):1403-10. doi:10.1007/s00125-004-1472-x.

6. Banz WJ, Maher MA, Thompson WG, Bassett DR, Moore W, Ashraf M, et al. Effects of resistance versus aerobic training on coronary artery disease risk factors. Exp Biol Med (Maywood). 2003;228(4):434-40.

7. Chodzko-Zajko WJ, Proctor DN, Fiatarone Singh MA, Minson CT, Nigg CR, Salem GJ, et al. American College of Sports Medicine position stand. Exercise and physical activity for older adults. Med Sci Sports Exerc. 2009;41(7):1510-30. doi:10.1249/ MSS.0b013e3181a0c95c.

8. Nelson ME, Rejeski WJ, Blair SN, Duncan PW, Judge JO, King AC, et al. Physical activity and public health in older adults: recommendation from the American College of Sports Medicine and the American Heart Association. Circulation. 2007;116(9):1094-105. doi:10.1161/ CIRCULATIONAHA.107.185650.

9. Stewart KJ, Bacher AC, Turner K, Lim JG, Hees PS, Shapiro EP, et al. Exercise and risk factors associated with metabolic syndrome in older adults. Am J Prev Med. 2005;28(1):9-18. doi:10.1016/j.amepre.2004.09.006.

10. Sillanpaa E, Hakkinen A, Punnonen K, Hakkinen K, Laaksonen DE. Effects of strength and endurance training on metabolic risk factors in healthy 40-65-yearold men. Scand J Med Sci Sports. 2009;19(6):885-95. doi:10.1111/j.1600-0838.2008.00849.x. 
11. Bartlett JD, Close GL, Maclaren DP, Gregson W, Drust B, Morton JP. High-intensity interval running is perceived to be more enjoyable than moderate-intensity continuous exercise: implications for exercise adherence. J Sports Sci. 2011;29(6):547-53. doi:10.1080/0 2640414.2010.545427.

12. Pardini R, Matsudo S, Araújo T, Matsudo V, Andrade E, Braggion G, et al. Validação do questionário internacional de nível de atividade física (IPAQ - versão 6): estudo piloto em adultos jovens brasileiros. RBCM. 2001;9(3):45-51.

13. Florindo AA, Latorre MRDO. Validação e reprodutibilidade do questionário de Baecke de avaliação da atividade física habitual em homens adultos. Rev Bras Med Esporte. 2003;9(3):121-8.

14. Libardi CA, Souza GV, Cavaglieri CR, Madruga VA, Chacon-Mikahil MP. Effect of resistance, endurance, and concurrent training on TNF-alpha, IL-6, and CRP. Med Sci Sports Exerc. 2012;44(1):50-6. doi:10.1249/ MSS.0b013e318229d2e9.

15. Libardi CA, Souza GV, Gaspari AF, dos Santos CF, Leite ST, Dias R, et al. Effects of concurrent training on interleukin-6, tumour necrosis factor-alpha and C-reactive protein in middle-aged men. J Sports Sci. 2011;29(14):1573-81. doi:10.1080/02640414.2011 .609896 .

16. Gordon CC, Chumlea WC, Roche A. Anthropometric standardizing reference manual. Champaign: Human Kinetics; 1988. p. 3-8.

17. Heyward VH. ASEP methods recommendation: body composition assessment. JEPonline. 2001;4:1-12.

18. Friedewald WT, Levy RI, Fredrickson DS. Estimation of the concentration of low-density lipoprotein cholesterol in plasma, without use of the preparative ultracentrifuge. Clin Chem. 1972;18(6):499-502. PMid:4337382.

19. Mancia G. Effective ambulatory blood pressure control in medical practice: good news to be taken with caution. Hypertension. 2007;49(1):17-8. doi:10.1161/01. HYP.0000250560.27738.72.

20. Brown LE, Weir JP. Procedures recommendation I: accurate assessment of muscular strength and power. JEPonline. 2001;4:1-21.

21. Jones AM, Doust JH. A 1\% treadmill grade most accurately reflects the energetic cost of outdoor running. J Sports Sci. 1996;14(4):321-7. doi:10.1080/026404196367796.
22. Wasserman K, McIlroy MB. Detecting the threshold of anaerobic metabolism in cardiac patients during exercise. Am J Cardiol. 1964;14:844-52. doi:10.1016/0002-9149(64)90012-8.

23. Heubert RA, Billat VL, Chassaing P, Bocquet V, Morton $\mathrm{RH}$, Koralsztein JP, et al. Effect of a previous sprint on the parameters of the work-time to exhaustion relationship in high intensity cycling. Int J Sports Med. 2005;26(7):583-92. doi:10.1055/s-2004-830335.

24. Howley ET, Bassett DR Jr., Welch HG. Criteria for maximal oxygen uptake: review and commentary. Med Sci Sports Exerc. 1995;27(9):1292-301. doi:10.1249/00005768-199509000-00009.

25. Sillanpaa E, Laaksonen DE, Hakkinen A, Karavirta L, Jensen B, Kraemer WJ, et al. Body composition, fitness, and metabolic health during strength and endurance training and their combination in middle-aged and older women. Eur J Appl Physiol. 2009;106(2):285-96. doi:10.1007/ s00421-009-1013-x.

26. Karavirta L, Hakkinen A, Sillanpaa E, Garcia-Lopez D, Kauhanen A, Haapasaari A, et al. Effects of combined endurance and strength training on muscle strength, power and hypertrophy in 40-67-year-old men. Scand J Med Sci Sports. 2011;21(3):402-11. doi:10.1111/j.1600-0838.2009.01059.x.

27. Sillanpaa E, Hakkinen A, Nyman K, Mattila M, Cheng S, Karavirta L, et al. Body composition and fitness during strength and/or endurance training in older men. Med Sci Sports Exerc. 2008;40(5):950-8. doi:10.1249/ MSS.0b013e318165c854.

28. Wijndaele K, Duvigneaud N, Matton L, Duquet W, Thomis M, Beunen G, et al. Muscular strength, aerobic fitness, and metabolic syndrome risk in flemish adults. Med Sci Sports Exerc. 2007;39(2):233-40. doi:10.1249/01. mss.0000247003.32589.a6.

29. Lemieux I, Pascot A, Couillard C, Lamarche B, Tchernof A, Almeras N, et al. Hypertriglyceridemic waist: a marker of the atherogenic metabolic triad (hyperinsulinemia; hyperapolipoprotein B; small, dense LDL) in men? Circulation. 2000;102(2):179-84. doi:10.1161/01. CIR.102.2.179.

30. Mattison R, Jensen M. The adipocyte as an endocrine cell. Curr Opin Endocrinol Diabetes. 2003;10(5):317-21. doi:10.1097/00060793-200310000-00002.

31. Tambalis K, Panagiotakos DB, Kavouras SA, Sidossis LS. Responses of blood lipids to aerobic, resistance, and combined aerobic with resistance exercise training: a systematic review of current evidence. Angiology. 2009;60(5):614-32. doi:10.1177/0003319708324927. 
32. Verney J, Kadi F, Saafi MA, Piehl-Aulin K, Denis C. Combined lower body endurance and upper body resistance training improves performance and health parameters in healthy active elderly. Eur J Appl Physiol. 2006;97(3):288-97. doi:10.1007/s00421-006-0175-z.

33. Shaw I, Shaw BS. Relationship between resistance training and lipoprotein profiles in sedentary male smokers. Cardiovasc J Afr. 2008;19(4):194-7. PMid:18776961.

34. Durstine JL, Grandjean PW, Davis PG, Ferguson MA, Alderson NL, DuBose KD. Blood lipid and lipoprotein adaptations to exercise: a quantitative analysis. Sports Med. 2001;31(15):1033-62. doi:10.2165/00007256200131150-00002.

35. Lewington S, Clarke R, Qizilbash N, Peto R, Collins R. Age-specific relevance of usual blood pressure to vascular mortality: a meta-analysis of individual data for one million adults in 61 prospective studies. Lancet. 2002;360(9349):1903-13. doi:10.1016/ S0140-6736(02)11911-8.

36. Keese F, Farinatti P, Pescatello L, Cunha FA, Monteiro WD. Aerobic exercise intensity influences hypotension following concurrent exercise sessions. Int J Sports Med. 2012;33(2):148-53. doi:10.1055/s-0031-1291321.
37. Cornelissen VA, Fagard RH. Effects of endurance training on blood pressure, blood pressure-regulating mechanisms, and cardiovascular risk factors. Hypertension. 2005;46(4):667-75. doi:10.1161/01. HYP.0000184225.05629.51.

38. Ghahramanloo E, Midgley AW, Bentley DJ. The effect of concurrent training on blood lipid profile and anthropometrical characteristics of previously untrained men. J Phys Act Health. 2009;6(6):760-6. PMid:20101919.

39. Haskell WL, Lee IM, Pate RR, Powell KE, Blair SN, Franklin BA, et al. Physical activity and public health: updated recommendation for adults from the American College of Sports Medicine and the American Heart Association. Med Sci Sports Exerc. 2007;39(8):1423-34. doi:10.1249/mss.0b013e3180616b27.

40. 40. Nelson ME, Rejeski WJ, Blair SN, Duncan PW, Judge JO, King AC, et al. Physical activity and public health in older adults: recommendation from the American College of Sports Medicine and the American Heart Association. Med Sci Sports Exerc. 2007;39(8):1435-45. doi:10.1249/mss.0b013e3180616aa2.

Recebido: $29 / 03 / 2012$

Received: 03/29/2012

Aprovado: 19/07/2012

Approved: 07/19/2012 